\title{
Evaluation of a program using a physician assistant and an electronic patient-provider communication tool to facilitate discussions about goals of care in older adults in hospital: a pilot study
}

\author{
Monica Monchis BScHons BScPA, Chris Martin MD, Giulio DiDiodato MD MPH PhD
}

\section{Abstract}

Background: Many patients receive unwanted, low-value, high-intensity care at the end of life because of poor communication with health care providers. Our aim was to evaluate the feasibility of using a physician assistant and an electronic tool to facilitate discussions about goals of care.

Method: We conducted a pilot study for the intervention (physician assistant-led discussion using an electronic tool) from Apr. 1 to Aug. 31, 2019. Patients aged 79 years or older admitted to the Royal Victoria Hospital (Barrie, Ontario) with either (i) no documented resuscitation preferences or (ii) a request for life-sustaining treatments in the event of a life-threatening illness were eligible for the intervention. The goal of this study was to complete more than 30 interventions. The primary outcomes included the proportion of consenting eligible patients, the time required and the proportion of patients changing their resuscitation preferences.

Results: A total of 763 patients met the inclusion criteria, with 337 eligible for the intervention. Of these, 49 cases were contacted for consent, and 37 interventions were completed (75.5\%, 95\% confidence interval [Cl] 61.1\%-86.6\%). On average, the intervention required 50 minutes (standard deviation 21) to complete. Overall, 31 interventions resulted in a change in resuscitation preferences (83.7\%, 95\% Cl $68.0 \%-93.8 \%)$, with 22 patients choosing to forgo any access to life-sustaining treatments in the event of a lifethreatening illness $(59.4 \%, 95 \% \mathrm{Cl} 42.1 \%-75.2 \%)$.

Interpretation: In this pilot study, the intervention was completed in a minority of eligible patients and required substantial time; however, it led to many changes in resuscitation preferences. Before designing a study to evaluate its impact, the intervention needs to be revised to make it more efficient to administer.

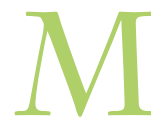
ore than $75 \%$ of patients admitted to hospitals with life-threatening illnesses lack decisionmaking capacity, yet only a minority will have communicated their treatment preferences with either their substitute decision-makers $(\mathrm{SDMs})^{1}$ or health care providers. ${ }^{2}$ As a result, up to 1 in 8 patients who die in hospital may have received low-value end-of-life care that was discordant with their goals. ${ }^{3-5}$

Goals-of-care discussions have been advocated to ensure that the wishes of patients admitted to hospitals for their current treatment preferences are known by their SDMs and health care providers. ${ }^{6}$ These discussions target patients admitted to hospital with or at a high risk of developing life-threatening illnesses. ${ }^{7}$ Although these discussions are not intended to elicit a "code status," they usually lead to the completion of physician order forms for life-sustaining treatments. ${ }^{8}$

These forms document treatment decisions for cardiopulmonary resuscitation (CPR) and life-sustaining treatments in the event of a cardiorespiratory arrest or other life-threatening illness. Although not considered to be part of a goals-of-care discussion, these forms have been shown to result in an improved degree of concordance between end-of-life care and resuscitation preferences. ${ }^{9,10}$

Most hospitals support the use of these resuscitation forms. However, most forms do not document the quality or content of the discussions about goals of care, bringing into question how informed these resuscitation decisions really are. ${ }^{11}$ In response, different tools have been developed to facilitate and

\section{Competing interests: None declared.}

This article has been peer reviewed.

Correspondence to: Giulio DiDiodato, didiodatog@rvh.on.ca CMAJ Open 2020. DOI:10.9778/cmajo.20200022 
standardize discussions about goals of care..$^{8,12-19}$ Most of these tools are disease specific and used in the outpatient setting, having limited applicability for a general hospital population. Outcomes-based research is scant, with mixed results to suggest that these tools improve patient-relevant or health care system outcomes. ${ }^{8}$

In this pilot study, our aim was to evaluate the feasibility of using a physician assistant (PA) and a locally developed electronic tool (e-tool) to facilitate discussions about goals of care by describing the number of eligible patients who completed discussions about goals of care, the time required to complete them and the resulting changes in their resuscitation decisions. In patients admitted to hospital and aged 79 years or older, we hypothesized that exposure to PA-led, e-tool-facilitated discussions about goals of care would result in less aggressive resuscitation preferences compared with their pre-exposure preferences.

\section{Methods}

\section{Study design}

This study is a nonrandomized pilot study enrolling consecutive patients starting on Apr. 1, 2019. We used the resuscitation preferences of a contemporaneous age-adjusted cohort as a control for comparative analyses.

\section{Setting and patients}

We recruited patients from the Royal Victoria Regional Health Centre, a 339-bed acute care community hospital located in Barrie, Ontario, starting on Apr. 1, 2019. We used flat sample size rules of thumb to estimate that we needed to complete at least 30 discussions to ensure a sufficient sample size to estimate the primary outcomes. ${ }^{20}$

The inclusion criteria were as follows: patients were aged 79 years or older; ${ }^{7}$ they had been in hospital for a period of more than 24 hours but less than 48 hours; physician order forms for life-sustaining treatments had either not been completed or included any of the following preferences: CPR, invasive life-sustaining treatments or noninvasive life-sustaining treatments (Appendix 1, available at www.cmajopen.ca/ content/8/3/E577/suppl/DC1); the patients spoke English or had a translator present; and the patients were competent or had an SDM.

Patients aged 79 years or older were enrolled because they accounted for more than $50 \%$ of all deaths at our hospital (calendar year 2018; data source: Decision Support Unit, Royal Victoria Regional Health Centre). In addition, studies suggest that these patients may not benefit from an intensive care unit (ICU) admission during a life-threatening illness. ${ }^{21-23}$

All patients who met the inclusion criteria were reviewed for any of the following exclusion criteria: the patient had received a new diagnosis of life-limiting illness during this hospital admission; the patient was clinically unstable or admitted to a high-intensity care unit; hospital discharge was planned within the next 24 hours; or the date for discussions about goals of care fell on a weekend.

\section{Program components and processes}

The Goals of Care program was developed by the investigators (G.D., C.M., M.M.) (Figure 1). The processes included identifying at-risk patients, securing consent, reviewing understanding of current illness and resuscitation preferences and their outcomes, discussing goals of care and their alignment with outcomes, and communicating resuscitation decisions. The components included an ICU physician assistant (M.M.), a locally developed electronic tool and a supervising intensivist (G.D., C.M.).

Before starting the pilot study, the investigators (G.D., C.M.) secured support for the program from all affected department chiefs, the chief of staff and hospital senior leadership. In addition, we conducted educational sessions for all affected nursing staff before program rollout (G.D., M.M.).

\section{Electronic tool}

The e-tool was developed (G.D.) using off-the-shelf software (FilemakerPro v14, www.filemaker.com/products/filemaker -pro-advanced/version-comparison.html\#fm14). We derived the contents of the e-tool from validated instruments or prognostic scoring tools ${ }^{19,24-27}$ (Appendix 1), and based them on the framework recommended by Hospice Palliative Care

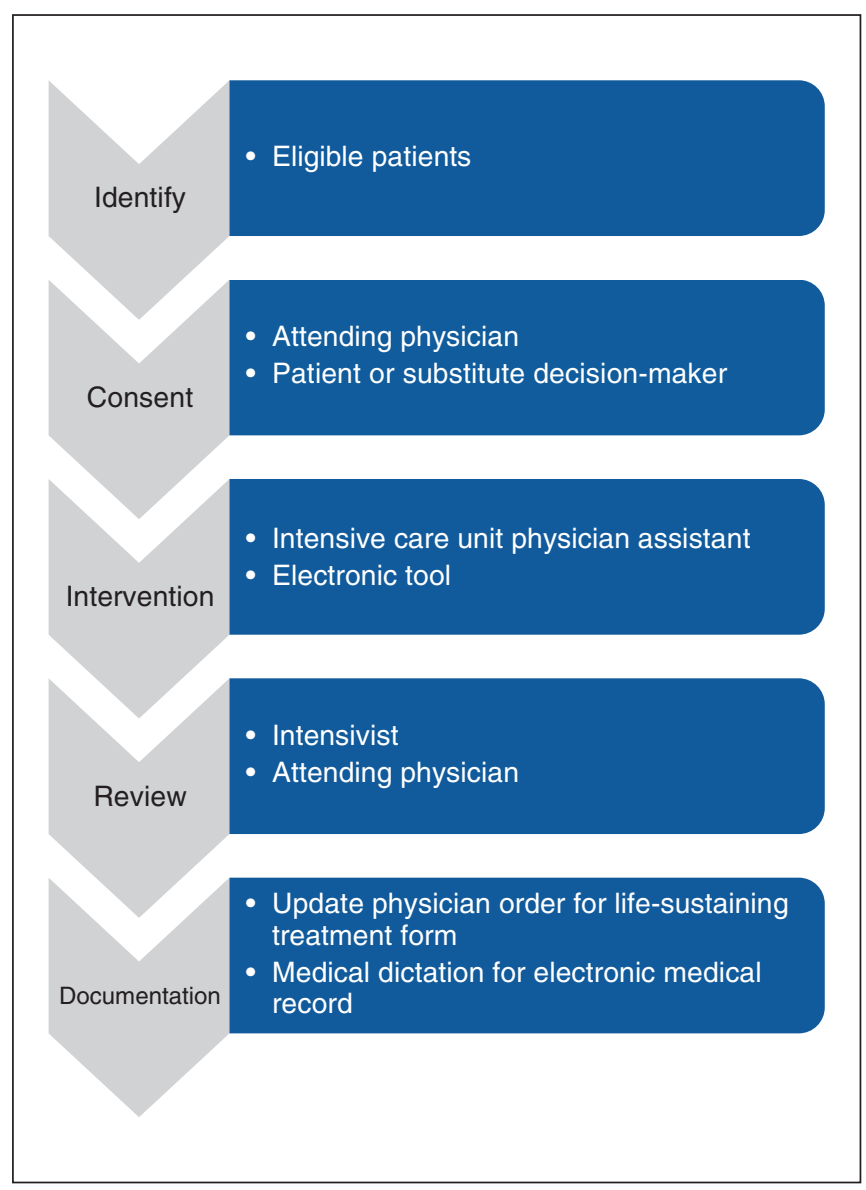

Figure 1: Components and processes associated with the Goals of Care program intervention (standardized approach to discussions about goals of care). 
Ontario. ${ }^{28}$ The criteria for choosing the individual components of the e-tool were that they were aligned to subject matter, disease agnostic, relevant to end-of-life discussions, generalizable across different populations and settings, simple to understand, took minimal time to complete, and easy to program into FileMakerPro (Appendix 1).

We beta-tested the e-tool on 31 patients in hospital from May 2018 to August 2018 to ensure stable Wi-Fi connectivity and valid data transmission. We asked these volunteers to provide informal feedback about the e-tool. Most recommendations focused on the display of information; most testers specifically preferred pictograms compared with other modes for the visual display of prognostic information.

\section{Physician assistant}

An ICU physician assistant (M.M.) was responsible for all the goals-of-care discussions during the pilot study. She was trained to use the e-tool by an ICU physician (G.D.). Having a physician assistant responsible for goals-of-care discussion was a precedent ${ }^{29}$ for our hospital, so the decision was based on the following factors: critical care expertise, time and flexibility, and maintenance and sustainability of the program.

Goals-of-care discussions require knowledge about critical care treatments, such as vasopressors and mechanical ventilation, along with their benefits and risks, and prognoses regarding morbidity and mortality. ${ }^{30,31}$ Most physicians identify this lack of expertise as a barrier to conducting these discussions. ${ }^{8,23,32}$ Discussions about goals of care require substantial time to organize and complete, ${ }^{33}$ and the PA had the most flexibility and time to commit to the study. We believed that the PA-led program model would be the most acceptable clinically and cost-effective approach at our institution.

Training of the PA occurred in the month preceding the start of the study. An ICU registered nurse who had been involved in the development and initial beta testing of the e-tool was responsible for training. A training schedule was developed that required the PA to observe between 3 and 5 e-tool-facilitated discussions conducted by the ICU nurse, followed by the PA conducting 3 to 5 e-tool-facilitated discussions under direct observation by the ICU nurse. After the PA had conducted 4 discussions, the ICU nurse deemed the PA ready to conduct the discussions independently.

\section{Consent and intervention}

On a daily basis, the PA ran a report, using the electronic medical record system, that identified all eligible patients. The PA reviewed each patient for any exclusion criteria and contacted each eligible patient's most responsible physician for permission to approach the patient or SDM. Once permission was secured from the physician, the PA used a scripted approach for consent from the patient or SDM to conduct a discussion about goals of care (Appendix 1). If consent was provided by the patient to contact the SDM, the PA contacted the patient or SDM to schedule a time that would accommodate the patient or both the patient and SDM being present for the discussion, where applicable. If the patient lacked capacity, the PA contacted the SDM to schedule a discussion about goals of care with them on behalf of the patient.

Every day, the PA attempted to contact all eligible patients. If the PA could not do so, those patients were not added to the next day's list. Instead, the PA documented that there was not sufficient time to conduct the discussion. This was done to determine the time and human resources that might be required to sustain such a program model in the future. The PA also prospectively documented other reasons for not completing a discussion about goals of care from a list that was defined a priori.

For patients with an SDM, the PA conducted discussions with both the patient and SDM present, where possible, to ensure that the SDM was aware of the patient's goals of care and informed treatment decisions. ${ }^{32,34}$ In this study, SDM refers to a person(s) who is authorized to provide or refuse consent to a treatment on behalf of a patient who lacks capacity as defined in the Health Care Consent Act. ${ }^{35}$

\section{Review and documentation}

Upon completing the discussion, the PA reviewed the patient's physician orders for life-sustaining treatment form with the patient or SDM, where applicable. For those patients with previously completed forms, the PA reviewed their original treatment decisions. For those patients or SDMs who wanted to change their form, the PA helped them complete a new form. For those patients or SDMs who had not previously completed a form, the PA explained the rationale and contents of the form and offered to help them complete the form at that time or any other time during their stay in hospital.

Once completed, an intensivist (G.D., C.M.) reviewed the discussions and their outcomes. After review, the PA contacted the most responsible physician to notify them of any changes in the patient's resuscitation decisions. The PA dictated a consult note using a standardized template (Appendix 1). This report was immediately available in the electronic medical record for review by any health care provider.

\section{Outcomes}

The investigators (G.D., C.M., M.M.) chose the primary outcomes to evaluate the feasibility and characteristics of the program and included the following: proportion of patients who did not consent to a discussion, proportion of eligible patients with a completed discussion, time required to complete a discussion, frequency of changes in resuscitation decisions and direction of changes in resuscitation decisions.

\section{Data collection}

We used the e-tool to collect data prospectively. The data were not stored on the mobile computer but were transmitted over an encrypted network to a server that was compliant with the Personal Health Information Protection Act (www.ontario.ca/ laws/statute/04p03). We collected patient data from the electronic medical record and from the patient or SDMs after obtaining consent (M.M.). We considered patients or SDMs who consented and completed the intervention to have been exposed. 


\section{Statistical analyses}

We used descriptive statistics to summarize the primary outcomes as proportions and means. We used confidence intervals (CIs) and standard deviations (SDs) to describe variance in proportions and means, respectively. We undertook comparative analysis between patients who were exposed and not exposed to the intervention using both logistic regression (reported as odds ratios [ORs] and 95\% CI) and difference in proportions (reported as percentage and 95\% CI). We used STATA/MP 15.0 for Mac (www.stata.com) for all statistical analyses.

\section{Ethics approval}

The Royal Victoria Regional Health Centre Research Ethics Board approved the pilot study on Feb. 11, 2019 (REB\#R18-028).

\section{Results}

From Apr. 1 to Aug. 31, 2019, 763 patients met the inclusion criteria (Figure 2), with a median of 5 patients (interquartile range 4) per day (range 1-16). After screening, exclusion and contact, 37 patients consented and completed the intervention (Table 1). This represents a 75.5\% (95\% CI 61.1\%-86.6\%) consent proportion among 49 cases contacted.

The most common reason for not completing discussions was a lack of time. On average, the discussions required

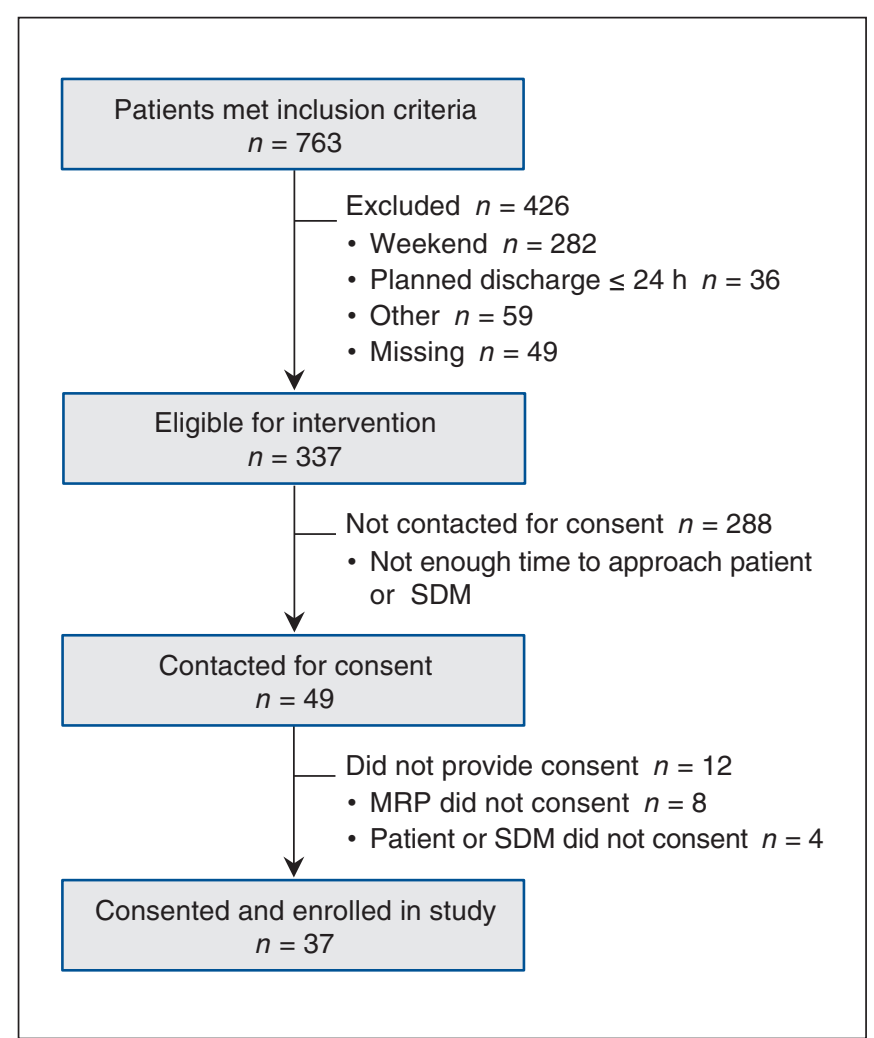

Figure 2: Patient flow diagram. Note: MRP = most responsible physician, SDM = substitute decision-maker, Missing = missing data for reason for exclusion.
50 minutes (SD 21 minutes) to complete. The SDMs were available for 36 (97.3\%, 95\% CI 85.8\%-99.9\%) discussions; 1 patient did not have an SDM. Compared with $511(70.4 \%$, 95\% CI 66.9\%-73.7\%) nonexposed patients, 30 (81.1\%, 95\% CI $64.8 \%-92.0 \%$ ) exposed patients consented to a less aggressive resuscitation plan. The distribution of resuscitation level preferences among those exposed and not exposed to the intervention is presented in Table 2 .

Compared with nonexposed patients, an absolute difference of $38.2 \%$ (95\% CI $22.1 \%-54.3 \%)$ fewer exposed patients chose resuscitation preferences that included admission to an ICU or high-dependency unit on their final form (Table 3).

\section{Interpretation}

We developed a multidimensional, disease-agnostic e-tool to facilitate discussions about goals of care in older patients admitted to the hospital. In this pilot study, about $11 \%$ of eligible patients completed a goals-of-care discussion using the e-tool. On average, this required about 50 minutes to complete. In this group, $60 \%$ chose to forgo any life-sustaining treatments compared with $21 \%$ in the nonexposed group.

The time required to schedule and complete these discussions was a substantial barrier leading to a substantial number of missed opportunities, an observation reported in other studies. ${ }^{16,37,38}$ Despite this, every patient or SDM who consented to a discussion completed the intervention. Among these exposed patients, many revised their resuscitation decisions to exclude life-sustaining treatments and CPR, a finding observed in other studies after accounting for sex, race and disease. ${ }^{39-42}$

For future use, we are modifying the e-tool to permit patients or SDMs to complete sections independently. The PA will then complete the prognostic scores with the patients or SDMs, along with explaining the resuscitation treatment options. These sections are complex and benefit from the involvement of a critical care expert to be informed. ${ }^{43}$

In an attempt to ensure that SDMs, where applicable, were present for discussions, we lost many opportunities as a result of the extra time needed to contact and schedule these discussions. In the future, nurses will organize these discussions with the patient or SDM and use a self-scheduling module we developed to notify the PA. We plan on expanding the inclusion criteria to include other high-risk populations, such as younger patients who have end-stage, life-limiting end-organ dysfunction.

\section{Limitations}

We included only patients aged 79 years or older, and thus it is unclear whether this process would be acceptable in other high-risk patient populations. We did not include any patients whose resuscitation decisions were either supportive or comfort care, and therefore could not determine whether these decisions were informed or could have been subject to change by exposure to the intervention. We did not have a comparator group a priori, so any differences 
Table 1 (part 1 of 2): Baseline characteristics of patients completing discussions about goals of care $(n=37)$

Baseline characteristic

Age, yr, mean $\pm S D$

Sex (female:male patients)

Residence

Community

Long-term care facility

Retirement home

"QoL in last two weeks?"25 $†$

Not answered

Very good

Good

Neither

Poor

Very poor

"Satisfied with health?"25 $†$

Not answered

Very satisfied

Satisfied

Neither

Dissatisfied

Very dissatisfied

Clinical frailty score, mean $\pm \mathrm{SD}^{24}$

Charlson Comorbidity Index score, mean $\pm \mathrm{SD}^{36}$

Expected hospital standardized mortality ratio, mean $\pm \mathrm{SD}^{26}$

Admission diagnoses ${ }^{26}$

Pneumonia

Fracture of femur

Sepsis

Heart failure

Acute renal failure

Unspecified dementia

Otherł

Most responsible physician

Hospitalist

Internal medicine specialist (or internist)

Surgeon (all types)

Hematology and oncology specialist

"Avoid attachment to machines?"19,34§

Not answered

10 (very important)

8

7

6

5

4

Unsure
No. (\%) of patients*

$86.7 \pm 4.7$

$16: 21$

$32(86.5)$

$3(8.1)$

$2(5.4)$

14 (37.8)

7 (18.9)

$12(32.4)$

$1(2.7)$

$2(5.4)$

$1(2.7)^{\star *}$

$14(37.8)$

2 (5.4)

12 (32.4)

2 (5.4)

6 (16.2)

$1(2.7)^{\text {** }}$

$4.5 \pm 1.8$

$4.6 \pm 3.4$

$29.8 \pm 14.0$

8 (21.6)

7 (18.9)

4 (10.8)

$3(8.1)$

2 (5.4)

2 (5.4)

$11(29.7)^{\star \star}$

12 (32.4)

14 (37.8)

9 (24.3)

$2(5.4)^{\star \star}$

17 (45.9)

7 (18.9)

1 (2.7)

2 (5.4)

$1(2.7)$

$6(16.2)$

1 (2.7)

$2(5.4)$ 
Table 1 (part 2 of 2): Baseline characteristics of patients completing discussions about goals of care $(n=37)$

\begin{tabular}{|c|c|}
\hline Baseline characteristic & No. $(\%)$ of patients* \\
\hline \multicolumn{2}{|l|}{ "Preserve life at all costs?"19,31§ } \\
\hline Not answered & $17(45.9)$ \\
\hline 10 (very important) & $3(8.1)$ \\
\hline 9 & $1(2.7)$ \\
\hline 8 & $2(5.4)$ \\
\hline 5 & $2(5.4)$ \\
\hline 4 & $2(5.4)$ \\
\hline 3 & $2(5.4)$ \\
\hline 2 & $2(5.4)$ \\
\hline 1 (not important) & $5(13.5)$ \\
\hline Unsure & $1(2.7)$ \\
\hline \multicolumn{2}{|c|}{ Physician order form for life-sustaining treatment status } \\
\hline Not completed & $22(59.4)$ \\
\hline Invasive and CPR & $11(29.7)$ \\
\hline Invasive and no CPR & $1(2.7)$ \\
\hline Minimally invasive and no CPR & $3(8.1)$ \\
\hline \multicolumn{2}{|c|}{$\begin{array}{l}\text { Note: CPR = cardiopulmonary resuscitation, SD = standard deviation, SDM = substitute decision-maker, QoL = quality of life. } \\
\text { *Unless otherwise specified. } \\
\text { †Based on patient or SDM response to: "How would you rate your quality of life in the last } 2 \text { weeks prior to admission to hospital?" or "How } \\
\text { satisfied are you with your health in the last } 2 \text { weeks prior to admission to hospital?", respectively. } \\
\text { fEleven diagnoses with a frequency of } 1 . \\
\text { §Based on patient or SDM response to "How important is it that I avoid being attached to machines and tubes?" or "How important is the belief } \\
\text { that life should be preserved at all costs?", respectively. } \\
\text { qlf physician order form for life-sustaining treatment status is not completed in prediscussions about goals of care, treatment of life-threatening } \\
\text { illnesses defaults to invasive and CPR. }\end{array}$} \\
\hline
\end{tabular}

Table 2: Distribution of pre- (within first $48 \mathrm{hr}$ of admission) and post- (at the time of discharge or death) resuscitation decisions documented on physician order forms in exposed and nonexposed groups

\begin{tabular}{|c|c|c|c|c|}
\hline \multirow[b]{2}{*}{ Resuscitation status } & \multicolumn{2}{|c|}{$\begin{array}{c}\text { No. }(\%) \text { of exposed patients } \\
\qquad n=37\end{array}$} & \multicolumn{2}{|c|}{$\begin{array}{l}\text { No. (\%) of nonexposed patients } \\
\qquad n=726\end{array}$} \\
\hline & Pre- & Post- & Pre- & Post- \\
\hline Not completed* & $22(59.4)$ & $11(29.7)$ & $108(14.9)$ & $15(2.0)$ \\
\hline Invasive and CPR & $11(29.7)$ & $1(2.7)$ & $387(53.3)$ & $8(1.1)$ \\
\hline Invasive and no CPR & $1(2.7)$ & $2(5.4)$ & $13(1.8)$ & $327(45.0)$ \\
\hline Minimally invasive and no CPR & $3(8.1)$ & $1(2.7)$ & $218(30.0)$ & $222(30.6)$ \\
\hline Supportive care & 0 & $1(2.7)$ & 0 & $118(16.2)$ \\
\hline Comfort care & 0 & $21(56.8)$ & 0 & $36(4.9)$ \\
\hline
\end{tabular}

between the exposed and nonexposed patients is exploratory and hypothesis generating.

Using a program led by a PA may not be practicable or cost-effective compared with one led by another health care provider. The intervention was hospital based and may not be feasible in an outpatient setting. About $30 \%$ of exposed patients did not complete their physician order form for life- sustaining treatments before discharge, suggesting that the timing of the intervention may have been too early or they could have benefited from another discussion. Although the sample size was small, the observed effect size was large and will be helpful in sample size calculations for the subsequent randomized study. At this time, the e-tool was also available only in English. 


\section{Table 3: Final resuscitation decisions among exposed and nonexposed patients}

\begin{tabular}{|c|c|c|c|}
\hline \multirow[b]{2}{*}{ Variable } & \multicolumn{2}{|c|}{ Resuscitation decisions for life-sustaining treatments } & \multirow[b]{2}{*}{ Total } \\
\hline & $\begin{array}{c}\text { Yes* } \\
\text { no. (\%) }\end{array}$ & $\begin{array}{c}\text { Not } \\
\text { no. (\%) }\end{array}$ & \\
\hline Exposed & $15(40.5)$ & $22(59.4)$ & 37 \\
\hline Nonexposed & $572(78.7)$ & 154 (21.2) & 726 \\
\hline Total & 587 & 176 & 763 \\
\hline \multicolumn{4}{|c|}{$\begin{array}{l}\text { Note: CPR = cardiopulmonary resuscitation. } \\
\text { *Includes the following categories in the physician order form for life-sustaining treatments: not completed, invasive and CPR, invasive and no CPR, minimally invasive and } \\
\text { no CPR. } \\
\text { tIncludes only supportive or comfort care. }\end{array}$} \\
\hline
\end{tabular}

\section{Conclusion}

In this pilot study, we implemented a PA-led, e-tool-facilitated goals-of-care program for older patients in hospital. Most contacted patients or SDMs consented to the intervention, as did the attending physicians. On average, the intervention took almost an hour to complete. Among patients who completed a discussion, about $60 \%$ chose end-of-life treatment preferences that did not include life-sustaining treatments. The current version of the program was inefficient, missing most eligible patients. We expect that e-tool modifications will reduce the time needed to complete these discussions, allowing us to scale up the program and capture more high-risk patients. We plan to conduct a randomized clinical study to determine whether the addition of the modified e-tool results in different resuscitation treatment decisions compared with usual care without the e-tool.

\section{References}

1. Fried TR, Zenoni M, Iannone L, et al. Engagement in advance care planning and surrogates' knowledge of patients' treatment goals. 7 Am Geriatr Soc 2017;65:1712-8.

2. Simon JE, Ghosh S, Heyland D, et al. Evidence of increasing public participation in advance care planning: a comparison of polls in Alberta between 2007 and 2013. BM7 Support Palliat Care 2019;9:189-96.

3. Khandelwal N, Curtis JR, Freedman VA, et al. How often is end-of-life care in the United States inconsistent with patients' goals of care? 7 Palliat Med 2017;20:1400-4.

4. Wendler D, Rid A. Systematic review: the effect on surrogates of making treatment decisions for others. Ann Intern Med 2011;154:336-46.

5. Bekelman JE, Halpern SD, Blankart CR, et al. Comparison of site of death, health care utilization, and hospital expenditures for patients dying with cancer in 7 developed countries. 7AMA 2016;315:272-83.

6. Hospice PCO. Person-centred decision-making (PCDM): advance care planning, goals of care discussion and health care consent tool kit. Toronto: Speak Up Ontario; 2019. Available: www.speakupontario.ca/person-centred-decision-making/ (accessed 2020 Mar. 1).

7. Heyland DK. Failure to engage hospitalized elderly patients and their families in advance care planning. FAMA Intern Med 2013;173:778-87.

8. Myers J, Cosby R, Gzik D, et al. Provider tools for advance care planning and goals of care discussions: a systematic review. Am 7 Hosp Palliat Care 2018; 35:1123-32.

9. Collier J, Kelsberg G, Safranek S. Clinical inquiries: How well do POLST forms assure that patients get the end-of-life care they requested? 7 Fam Pract 2018;67:249-51.

10. Lee MA, Brummel-Smith K, Meyer J, et al. Physician orders for life-sustaining treatment (POLST): outcomes in a PACE program. Program of all-inclusive care for the elderly. 7 Am Geriatr Soc 2000;48:1219-25.

11. DiDiodato G. Estimating the impact of words used by physicians in advance care planning discussions: the "Do you want everything done?" effect. Crit Care Explor 2019;1:e052.

12. Austin CA, Mohottige D, Sudore RL, et al. Tools to promote shared decision-making in serious illness. FAMA Intern Med 2015;175:1213-21.
13. Edmonds KP, Ajayi TA. Do we know what we mean? An examination of the use of the phrase "Goals of Care" in the literature. 7 Palliat Med 2019; 22:1546-52.

14. Schiff R, Shaw R, Raja N, et al. Advance end-of-life healthcare planning in an acute NHS hospital setting; development and evaluation of the Expression of Healthcare Preferences (EHP) document. Age Ageing 2009;38:81-5.

15. Jain A, Corriveau S, Quinn K, et al. Video decision aids to assist with advance care planning: a systematic review and meta-analysis. BM7 Open 2015;5:e007491.

16. Mills AC, Levinson M, Dunlop WA, et al. Testing a new form to document "Goals-of-Care" discussions regarding plans for end-of-life care for patients in an Australian emergency department. Emerg Med Australas 2018;30:777-84.

17. Saunders CH, Patel K, Kang H, et al. Serious choices: a systematic environmental scan of decision aids and their use for seriously ill people near death. $\mathcal{F}$ Hosp Med 2019;14:294-302.

18. Littell RD, Kumar A, Einstein MH, et al. Advanced communication: a critical component of high-quality gynecologic cancer care: a Society of Gynecologic Oncology evidence-based review and guide. Gynecol Oncol 2019;155:161-9.

19. You JJ, Dodek P, Lamontagne F, et al. What really matters in end-of-life discussions? Perspectives of patients in hospital with serious illness and their families. CMA7 2014;186:E679-87.

20. Machin D, Campbell M, Tan S, et al. Sample size for clinical, laboratory and epidemiology studies. 4th ed. Hoboken (NJ): John Wiley \& Sons; 2018:266.

21. Guidet B, Leblanc G, Simon T, et al. Effect of systematic intensive care unit triage on long-term mortality among critically ill elderly patients in France. 7AMA 2017;318:1450-9.

22. Guidet B, Vallet H, Boddaert J, et al. Caring for the critically ill patients over 80: a narrative review. Ann Intensive Care 2018;8:114-28.

23. Hwe C, Parrish J, Berry B, et al. Nonbeneficial intensive care: misalignments between provider assessments of benefit and use of invasive treatments. F Intensive Care Med 2019 Jan 29. [Epub ahead of print]. doi: 10.1177/0885066619826044.

24. Rockwood K. A global clinical measure of fitness and frailty in elderly people. CMA7 2005;173:489-95.

25. Skevington SM, Lotfy M, O'Connell KA. The World Health Organization's WHOQOL-BREF quality of life assessment: psychometric properties and results of the international field trial. a report from the WHOQOL Group. Qual Life Res 2004;13:299-310.

26. Hospital standardized mortality ratio: technical notes. Ottawa: Canadian Institute for Health Information; 2019.

27. Harrison DA, Patel K, Nixon E, et al. Development and validation of risk models to predict outcomes following in-hospital cardiac arrest attended by a hospital-based resuscitation team. Resuscitation 2014;85:993-1000.

28. Approaches to goals of care discussions: resource for healthcare providers. Toronto: Ontario Palliative Care Network. Available: www.ontariopalliativecarenetwork.ca/sites/ opcn/files/Approaches'ToGoalsOfCare.pdf (accessed 2020 Apr. 1).

29. Dixon J, Knapp M. Whose job? The staffing of advance care planning support in twelve international healthcare organizations: a qualitative interview study. BMC Palliat Care 2018;17:78.

30. Prescott HC, Angus DC. Enhancing recovery from sepsis: a review. FAMA 2018;319:62-75.

31. Andersen LW, Holmberg MJ, Berg KM, et al. In-hospital cardiac arrest: a review. Vol. 321. 7AMA 2019;321:1200-10.

32. Scheunemann LP, Ernecoff NC, Buddadhumaruk P, et al. Clinician-family communication about patients' values and preferences in intensive care units. 7AMA Intern Med 2019;179:676-84.

33. Lund S, Richardson A, May C. Barriers to advance care planning at the end of life: an explanatory systematic review of implementation studies. PLoS One 2015;10:e116629.

34. Abdul-Razzak A, Heyland DK, Simon J, et al. Patient-family agreement on values and preferences for life-sustaining treatment: results of a multicentre observational study. BMJ Support Palliat Care 2019;9:e20. 
35. Health Care Consent Act 1996 p. SO 1996, c 2, Sch A. Available: www.canlii.org/ en/on/laws/stat/so-1996-c-2-sch-a/latest/so-1996-c-2-sch-a.html accessed 2020 Sept. 1).

36. Charlson ME, Pompei P, Ales KL, et al. A new method of classifying prognostic comorbidity in longitudinal studies: development and validation. $\mathcal{F}$ Chronic Dis 1987;40:373-3.

37. Bekelman DB, Johnson-Koenke R, Ahluwalia SC, et al. Development and feasibility of a structured goals of care communication guide. 7 Palliat Med 2017;20:1004-12.

38. Nair R, Kohen SA. Can a patient-directed video improve inpatient advance care planning? A prospective pre-post cohort study. BMF Qual Saf 2019;28:887-93. A

39. Mack JW, Cronin A, Keating NL, et al. Associations between end-of-life discussion characteristics and care received near death: a prospective cohort study. 7 Clin Oncol 2012;30:4387-95.

40. Zhang B, Wright AA, Nilsson ME, et al. Associations between advanced cancer patients' end-of-life conversations and cost experiences in the final week of life. 7 Clin Oncol 2008;26(15 Suppl):9530.

41. Sharma RK, Prigerson HG, Penedo FJ, et al. Male-female patient differences in the association between end-of-life discussions and receipt of intensive care near death. Cancer 2015;121:2814-20.

42. Loggers ET, Maciejewski PK, Paulk E, et al. Racial differences in predictors of intensive end-of-life care in patients with advanced cancer. 7 Clin Oncol 2009:27:5559-64.

43. Cardona-Morrell M, Benfatti-Olivato G, Jansen J, et al. A systematic review of effectiveness of decision aids to assist older patients at the end of life. Patient Educ Couns 2017;100:425-35.
Affiliations: Department of Critical Care Medicine (Monchis, Martin, DiDiodato), Royal Victoria Regional Health Centre, Barrie, Ont.; Department of Health Research Methods, Evidence and Impact (DiDiodato), McMaster University, Hamilton, Ont.

Contributors: Giulio DiDiodato created the e-tool. All authors designed the study, and were involved in the data collection, analyses and interpretation. Giulio DiDiodato drafted the article. Chris Martin and Monica Monchis reviewed and revised it critically for important content. All authors provided final approval of the article and agreed to act as guarantors of the work.

Data sharing: Data may be available from the corresponding author upon request.

Acknowledgements: The authors thank the Royal Victoria Hospital Foundation for supporting the publication costs of this manuscript. They also thank the Royal Victoria Regional Health Centre's Information Technology department and Decision Support Unit. In addition, they thank the following for their support during the pilot study: Sherry Hubbert, Anjolaoluwa Ogunsina, Aidan McKee, Devon Harvey, Niamh McKee.

Supplemental information: For reviewer comments and the original submission of this manuscript, please see www.cmajopen.ca/content/8/3/ E577/suppl/DC1. 